\title{
Introduction of the Tensor Which Satisfied Binary Law
}

\section{Koji Ichidayama}

716-0002 Okayama, Japan

Email: ichikoji@lime.ocn.ne.jp

How to cite this paper: Ichidayama, $\mathrm{K}$. (2017) Introduction of the Tensor Which Satisfied Binary Law. Journal of Modern Physics, 8, 126-132.

http://dx.doi.org/10.4236/jmp.2017.81011

Received: December 29, 2016

Accepted: January 20, 2017

Published: January 23, 2017

Copyright $\odot 2017$ by author and Scientific Research Publishing Inc. This work is licensed under the Creative Commons Attribution International License (CC BY 4.0).

http://creativecommons.org/licenses/by/4.0/ c) (i) Open Access

\begin{abstract}
P: For every coordinate system, there is no immediate reason for preferring certain systems of co-ordinates to others. If we don't recognize that $\mathrm{P}$ is establishment, we must recognize to existence of the absolute coordinate system. Therefore, we must recognize that $\mathrm{P}$ is establishment. Nevertheless, I got conclusion that $\mathrm{P}$ isn't establishment for all coordinate systems $x^{\mu}, x^{\nu}, x^{\sigma}, x^{\lambda}, \cdots$. If $\mathrm{P}$ is establishment, this is the trouble. As against, I got conclusion that if we consider "Binary Law" for all coordinate systems $x^{\mu}, x^{\nu}, x^{\sigma}, x^{\lambda}, \cdots, \mathrm{P}$ is establishment for all coordinate systems $x^{\mu}, x^{\nu}, x^{\sigma}, x^{\lambda}, \cdots$. If we consider Binary Law for all coordinate systems $x^{\mu}, x^{\nu}, x^{\sigma}, x^{\lambda}, \cdots$, we must consider Binary Law for the coordinate systems using into Tensor, too. So, I decided to report for the Tensor which satisfied Binary Law.
\end{abstract}

\section{Keywords}

Tensor, Covariant Derivative

\section{Introduction}

Definition 1. For every coordinate system, there is no immediate reason for preferring certain systems of co-ordinates to others.

Definition 2. I named $\overline{x^{\mu}} \neq x^{\mu}, \overline{x^{v}} \neq x^{\nu}, \overline{x^{\mu}}=x^{v}, \overline{x^{v}}=x^{\mu}$ "Binary Law".

Definition 3. $x^{\mu} \neq x_{\mu}$ is established.

Definition 4. $x^{v} \neq x_{v}$ is established.

Definition 5. $x^{\mu} \neq-x^{\mu}$ is established.

Definition 6. Convariant and contravariant tensor of the first rank $A_{\mu}, A_{v}, A^{\mu}, A^{\nu}$ satisfied $A_{\mu}=\frac{\partial x^{v}}{\partial x^{\mu}} A_{r}, A^{\mu}=\frac{\partial x^{\mu}}{\partial x^{v}} A^{v} \quad[1]$.

Definition 7. Tensor of rank zero $A_{\mu}^{\mu}, A_{v}^{v}$ satisfied $A_{\mu}^{\mu}=A_{v}^{v}$ [1].

Definition 8. If tensor $A_{\nu}^{\mu}$ satisfied $A_{v}^{\mu}=A_{\mu}^{v}$, this tensor $A_{r}^{\mu}$ was named symmetric tensor [1]. 
Definition 9. Convariant differentiation for Convariant Bector $A_{\mu ; \nu}$ satisfied $A_{\mu ; v}=\frac{\partial A_{\mu}}{\partial x^{v}}-\{\mu \nu, \tau\} A_{\tau}=\frac{\partial A_{\mu}}{\partial x^{v}}-A_{\tau} \frac{1}{2} g^{\tau \sigma}\left(\frac{\partial g_{\mu \sigma}}{\partial x^{v}}+\frac{\partial g_{v \sigma}}{\partial x^{\mu}}-\frac{\partial g_{\mu v}}{\partial x^{\sigma}}\right)[1]$.

Definition 10. $g_{\mu}^{\mu}=1$ and $g_{v}^{\mu}=0:(\mu \neq v)$ are establishment [2].

Definition 11. Convariant differentiation for contravariant bector $A_{, v}^{\mu}$ satisfied $A_{, v}^{\mu}=\frac{\partial A^{\mu}}{\partial x^{v}}+\{\tau v, \mu\} A^{\tau}=\frac{\partial A^{\mu}}{\partial x^{v}}+A^{\tau} \frac{1}{2} g^{\mu \sigma}\left(\frac{\partial g_{\tau \sigma}}{\partial x^{v}}+\frac{\partial g_{v \sigma}}{\partial x^{\tau}}-\frac{\partial g_{\tau v}}{\partial x^{\sigma}}\right)$ [2] .

Definition 12. Convariant differentiation for Scalar $S_{; v}$ satisfied $S_{; v}=\frac{\partial S}{\partial x^{v}} \quad[2]$.

\section{About Reason to Take Binary Law into Consideration}

We will have to receive existence of the absolute coordinate system if Definition 1 is not established. Therefore, we must accept establishment of Definition 1.

Proposition 1. Definition 1 is not established for all coordinate systems $x^{\mu}, x^{v}, x^{\sigma}, x^{\lambda}, \cdots$.

Proof: All coordinate systems $x^{\mu}, x^{\nu}, x^{\sigma}, x^{\lambda}, \cdots$ thinks about $x^{\mu}$ in a standard and can divide it into two next groups.

$$
\begin{gathered}
x^{\mu}=x^{\mu}, \\
x^{\mu} \neq x^{v}, x^{\mu} \neq x^{\sigma}, x^{\mu} \neq x^{\lambda}, \cdots .
\end{gathered}
$$

I think that I change the coordinate systems of the standard $x^{\mu}$ of (1) for all coordinate systems $x^{\mu}, x^{\nu}, x^{\sigma}, x^{\lambda}, \cdots$ sequentially now. By the way, the difference cannot occur between each conclusion to be provided here if Definition 1 is established. This reason is that all coordinate systems $x^{\mu}, x^{v}, x^{\sigma}, x^{\lambda}, \cdots$ has a privilege of the equality each other if Definition 1 is established. At first (1) gets an invariable conclusion for $\mu, \mu$ exchange. Therefore, at least (1) must get an invariable conclusion for the next $\mu, v$ exchange if Definition 1 is established. Here, I get

$$
\begin{gathered}
x^{v}=x^{v}, \\
x^{v} \neq x^{\mu}, x^{v} \neq x^{\sigma}, x^{v} \neq x^{\lambda}, \cdots
\end{gathered}
$$

by $\mu, v$ exchange from (1). Therefore, (2) must be equal with (1) if Definition 1 is established. By the way, $x^{\mu}=x^{\mu}, x^{\mu} \neq x^{v}$ of (1) is equal with $x^{v}=x^{v}, x^{v} \neq x^{\mu}$ of (2), but $x^{\mu} \neq x^{\sigma}, x^{\mu} \neq x^{\lambda}, \cdots$ of (1) is not equal with $x^{v} \neq x^{\sigma}, x^{v} \neq x^{\lambda}, \cdots$ of (2). In other words, (2) is not equal with (1). Therefore, Definition 1 is not established for all coordinate systems $x^{\mu}, x^{v}, x^{\sigma}, x^{\lambda}, \cdots$.

-End Proof

Establishment of Proposition 1 is a problem in thinking that Definition 1 must be established. Therefore, I aim at getting establishment of Definition 1 for all coordinate systems $x^{\mu}, x^{\nu}, x^{\sigma}, x^{\lambda}, \cdots$.

Proposition 2. If all coordinate systems $x^{\mu}, x^{v}, x^{\sigma}, x^{\lambda}, \cdots$ satisfies $\overline{x^{\mu}} \neq x^{\mu}, \overline{x^{v}} \neq x^{v}, \overline{x^{\mu}}=x^{v}, \overline{x^{v}}=x^{\mu}$, Definition 1 is established for all coordinate systems $x^{\mu}, x^{\nu}, x^{\sigma}, x^{\lambda}, \cdots$.

Proof: I get

$$
x^{\mu}=x^{\mu},
$$




$$
\begin{aligned}
& x^{\mu} \neq x^{v}, \\
& x^{v}=x^{v}, \\
& x^{v} \neq x^{\mu}
\end{aligned}
$$

from (1), (2) if all coordinate systems $x^{\mu}, x^{v}, x^{\sigma}, x^{\lambda}, \cdots$ satisfies

$$
\overline{x^{\mu}} \neq x^{\mu}, \overline{x^{v}} \neq x^{v}, \overline{x^{\mu}}=x^{v}, \overline{x^{v}}=x^{\mu} \text {. }
$$

(3) is equal with (4) here. In other words, (2) is equal with (1) if all coordinate systems $x^{\mu}, x^{v}, x^{\sigma}, x^{\lambda}, \cdots$ satisfies (5). Therefore, Definition 1 is established for all coordinate systems $x^{\mu}, x^{v}, x^{\sigma}, x^{\lambda}, \cdots$ if all coordinate systems $x^{\mu}, x^{v}, x^{\sigma}, x^{\lambda}, \cdots$ satisfies (5).

-End Proof

Proposition 3. If all coordinate systems $x^{\mu}, x^{v}, x^{\sigma}, x^{\lambda}, \cdots$ satisfies $\overline{x^{\mu}} \neq x^{\mu}, \overline{x^{v}} \neq x^{v}, \overline{x^{\mu}}=x^{v}, \overline{x^{v}}=x^{\mu}$, all coordinate systems $x^{\mu}, x^{v}, x^{\sigma}, x^{\lambda}, \cdots$ shifts to only two of $x^{\mu}, x^{v}$.

Proof: If all coordinate systems $x^{\mu}, x^{v}, x^{\sigma}, x^{\lambda}, \cdots$ satisfies (5), I get $x^{\mu}, x^{v}$ than all coordinate systems $x^{\mu}, x^{v}, x^{\sigma}, x^{\lambda}, \cdots$.

-End Proof

Proposition 4. If $\overline{x^{\mu}} \neq x^{\mu}, \overline{x^{v}} \neq x^{v}, \overline{x^{\mu}}=x^{v}, \overline{x^{v}}=x^{\mu}$ is established, $x^{\mu} \neq x^{v}$ is established.

Proof: I get

$$
\overline{x^{\mu}} \neq x^{\mu}, \overline{x^{v}} \neq x^{v}, \overline{x^{\mu}}=x^{\mu}, \overline{x^{v}}=x^{v}
$$

from (5), (7) if I assume establishment of

$$
x^{\mu}=x^{v}
$$

when (5) is established. Because (6) includes contradiction,

$$
x^{\mu} \neq x^{v}
$$

is established when (5) is established.

Proposition 5. If $\overline{x^{\mu}} \neq x^{\mu}, \overline{x^{v}} \neq x^{v}, \overline{x^{\mu}}=x^{v}, \overline{x^{v}}=x^{\mu}$ is established, $x^{v}=x_{\mu}, x^{\mu}=x_{v}, x^{v}=-x^{\mu}, x_{v}=-x_{\mu}$ are established.

Proof: When (5) is established, (8) is established from Proposition 4. Therefore, I get

$$
x^{\mu} \neq x_{\mu} \text { (False) }
$$

from (8), (10) if I assume establishment of $x^{v} \neq x_{\mu}$ when (5) is established. I can rewrite $x^{v} \neq x_{\mu}$ as

$$
\left.x^{v}=x_{\mu} \text { (False }\right)
$$

here. When (5) is established, I get

$$
x^{\mu} \neq x_{\mu}
$$

from Definition 3. Because (9) includes contradiction for (11),

$$
x^{v}=x_{\mu}
$$

is established when (5) is established.

Similary, I get 


$$
x^{v} \neq x_{v} \text { (False) }
$$

from (8), (14) if I assume establishment of $x^{\mu} \neq x_{v}$ when (5) is established. I can rewrite $x^{\mu} \neq x_{v}$ as

$$
x^{\mu}=x_{v} \text { (False) }
$$

here. When (5) is established, I get

$$
x^{v} \neq x_{v}
$$

from Definition 4. Because (13) includes contradiction for (15),

$$
x^{\mu}=x_{v}
$$

is established when (5) is established.

Similary, I get

$$
x^{\mu} \neq-x^{\mu} \text { (False) }
$$

from (8), (18) if I assume establishment of $x^{v} \neq-x^{\mu}$ when (5) is established. I can rewrite $x^{v} \neq-x^{\mu}$ as

$$
x^{v}=-x^{\mu}(\text { False })
$$

here. When (5) is established, I get

$$
x^{\mu} \neq-x^{\mu}
$$

from Definition 5. Because (17) includes contradiction for (19),

$$
x^{v}=-x^{\mu}
$$

is established when (5) is established. And, I get

$$
x_{v}=-x_{\mu}
$$

from (12), (16), (20).

-End Proof

\section{About the Tensor Which Satisfied Binary Law}

We will have to think about adaptation of the establishment of Binary Law for the coordinate systems $x^{\mu}, x^{v}, x^{\sigma}, x^{\lambda}, \cdots$ in the tensor if we think about establishment of Binary Law for all coordinate systems $x^{\mu}, x^{v}, x^{\sigma}, x^{\lambda}, \cdots$. Therefore, I decided to report Tensor when all coordinate systems $x^{\mu}, x^{v}, x^{\sigma}, x^{\lambda}, \cdots$ satisfied Binary Law.

Proposition 6. If all coordinate systems $x^{\mu}, x^{\nu}, x^{\sigma}, x^{\lambda}, \cdots$ satisfied $\overline{x^{\mu}} \neq x^{\mu}, \overline{x^{v}} \neq x^{v}, \overline{x^{\mu}}=x^{v}, \overline{x^{v}}=x^{\mu}$, Convariant and Contravariant Tensor of the first rank does not change the form of the equation.

Proof: I get

$$
A_{\mu}=\frac{\partial x^{v}}{\partial x^{\mu}} A_{\nu}, A^{\mu}=\frac{\partial x^{\mu}}{\partial x^{v}} A^{v}
$$

from Definition 6 if all coordinate systems $x^{\mu}, x^{v}, x^{\sigma}, x^{\lambda}, \cdots$ satisfies (5). Definition 6 and (22) are equal here. Therefore, if all coordinate systems $x^{\mu}, x^{\nu}, x^{\sigma}, x^{\lambda}, \cdots$ satisfied (5), Convariant and Contravariant Tensor of the first rank does not change the form of the equation. 
-End Proof

Proposition 7. Tensor of the second rank becomes Symmetric Tensor if all coordinate systems $x^{\mu}, x^{\nu}, x^{\sigma}, x^{\lambda}, \cdots$ satisfies $\overline{x^{\mu}} \neq x^{\mu}, \overline{x^{\nu}} \neq x^{\nu}, \overline{x^{\mu}}=x^{\nu}, \overline{x^{v}}=x^{\mu}$.

Proof: I get

$$
A_{\mu}^{\mu}=A_{\nu}^{v}
$$

from Definition 7 if all coordinate systems $x^{\mu}, x^{\nu}, x^{\sigma}, x^{\lambda}, \cdots$ satisfies (5). Definition 7 and (23) are equal here. We can use (12), (16), (20), (21) for (23) by considering Proposition 5 here. And we can rewrite (23) by using (12), (16) for

$$
\begin{aligned}
& A_{\nu \mu}=A_{\nu}^{v}, A^{\mu v}=A_{\nu}^{v}, \\
& A_{\mu}^{\mu}=A_{\mu v}, A_{\mu}^{\mu}=A^{v \mu} .
\end{aligned}
$$

Then, I get

$$
A_{\nu \mu}=A_{\mu v}, A^{\mu v}=A^{v \mu}
$$

from (23),(24). And we can rewrite (23) by using (20), (21) for

$$
-A_{\mu}^{v}=A_{\nu}^{v},-A_{\nu}^{\mu}=A_{\nu}^{v} .
$$

Then, I get

$$
A_{\mu}^{v}=A_{v}^{\mu}
$$

from (26). Therefore, Tensor of the second rank becomes Symmetric Tensor than consideration of Definition 8 when all coordinate systems $x^{\mu}, x^{\nu}, x^{\sigma}, x^{\lambda}, \cdots$ satisfies (5).

-End Proof

Proposition 8. If all coordinate systems $x^{\mu}, x^{\nu}, x^{\sigma}, x^{\lambda}, \cdots$ satisfied $\overline{x^{\mu}} \neq x^{\mu}, \overline{x^{v}} \neq x^{v}, \overline{x^{\mu}}=x^{v}, \overline{x^{v}}=x^{\mu}$, The distance of two points be able to change oneself in connection with the metric of space.

Proof: I get

$$
g_{\mu}^{\mu}=1, g_{v}^{\mu}=0:(\mu \neq v)
$$

from Definition 10 if all coordinate systems $x^{\mu}, x^{\nu}, x^{\sigma}, x^{\lambda}, \cdots$ satisfies (5). I get

$$
\begin{aligned}
A_{\mu ; \nu}=\frac{\partial A_{\mu}}{\partial x^{v}} & -A_{v} \frac{1}{2} g^{v v}\left(\frac{\partial g_{\mu v}}{\partial x^{v}}+\frac{\partial g_{v v}}{\partial x^{\mu}}-\frac{\partial g_{\mu v}}{\partial x^{v}}\right) \\
& =\frac{\partial A_{\mu}}{\partial x^{v}}-\frac{1}{2}\left(\frac{\partial g_{v}^{v}}{\partial x^{\mu}}\right) A_{\nu} \\
& =\frac{\partial A_{\mu}}{\partial x^{v}}-\frac{1}{2}\left(\frac{\partial g_{v}^{\sigma}}{\partial x^{\mu}}\right) A_{\sigma} \\
& =\frac{\partial A_{\mu}}{\partial x^{v}}-\frac{1}{2}\left(\frac{\partial g_{\sigma}^{\sigma}}{\partial x^{\mu}}\right) A_{v}
\end{aligned}
$$

from Definition 9 if all coordinate systems $x^{\mu}, x^{\nu}, x^{\sigma}, x^{\lambda}, \cdots$ satisfies (5). By the way, we cannot handle (30), (31) according to Proposition 3. We can use (12), (16), (20), (21) for (29) by considering Proposition 5 here. And we must rewrite (29) by using (16) for 


$$
\begin{aligned}
A_{\mu ;}^{\mu} & =\frac{\partial A_{\mu}}{\partial x_{\mu}}-\frac{1}{2}\left(\frac{\partial g^{v \mu}}{\partial x^{\mu}}\right) A \\
& =\frac{\partial A_{\mu}}{\partial x_{\mu}}-\frac{1}{2}\left(\frac{\partial g_{v}^{v}}{\partial x^{\mu}}\right) A^{\mu} .
\end{aligned}
$$

I decide not to handle (33) by consideration of (28) here. Well, I get conclution from (32) that if all coordinate systems $x^{\mu}, x^{\nu}, x^{\sigma}, x^{\lambda}, \cdots$ satisfied (5), Scalar quantity be able to change oneself in connection with the metric of space. Here, This Scalar quantity expressed the all of quantity expressed as Scalar. Therefore, I get conclution that the distance of two points be able to change oneself in connection with the metric of space.

-End Proof

Proposition 9. If all coordinate systems $x^{\mu}, x^{\nu}, x^{\sigma}, x^{\lambda}, \cdots$ satisfied $\overline{x^{\mu}} \neq x^{\mu}, \overline{x^{\nu}} \neq x^{\nu}, \overline{x^{\mu}}=x^{\nu}, \overline{x^{\nu}}=x^{\mu}$, convariant differentiation for Contravariant Bector $A_{, v}^{\mu}$ behave like a convariant differentiation for Scalar $S_{; v}$.

Proof: I get

$$
\begin{aligned}
A_{, v}^{\mu}=\frac{\partial A^{\mu}}{\partial x^{v}} & +A^{v} \frac{1}{2} g^{\mu v}\left(\frac{\partial g_{v v}}{\partial x^{v}}+\frac{\partial g_{v v}}{\partial x^{v}}-\frac{\partial g_{v v}}{\partial x^{v}}\right) \\
& =\frac{\partial A^{\mu}}{\partial x^{v}}+\frac{1}{2}\left(\frac{\partial g_{v}^{\mu}}{\partial x^{v}}\right) A^{v} \\
& =\frac{\partial A^{\mu}}{\partial x^{v}}+\frac{1}{2}\left(\frac{\partial g_{\sigma}^{\mu}}{\partial x^{v}}\right) A^{\sigma} \\
& =\frac{\partial A^{\mu}}{\partial x^{v}}+\frac{1}{2}\left(\frac{\partial g_{v}^{\mu}}{\partial x^{\sigma}}\right) A^{\sigma}
\end{aligned}
$$

from Definition 11 if all coordinate systems $x^{\mu}, x^{\nu}, x^{\sigma}, x^{\lambda}, \cdots$ satisfies (5). By the way, we cannot handle (35), (36) according to Proposition 3. We can use (12), (16), (20), (21) for (34) by considering Proposition 5 here. And we must rewrite (34) by using (21) for

$$
\begin{aligned}
-A_{, \mu}^{\mu} & =-\frac{\partial A^{\mu}}{\partial x^{\mu}}-\frac{1}{2}\left(\frac{\partial g_{v}^{\mu}}{\partial x^{\mu}}\right) A^{v} \\
& =-\frac{\partial A^{\mu}}{\partial x^{\mu}}-\frac{1}{2}\left(\frac{\partial g_{\mu}^{\mu}}{\partial x^{v}}\right) A^{v} .
\end{aligned}
$$

And, I can get

$$
-A_{, \mu}^{\mu}=-\frac{\partial A^{\mu}}{\partial x^{\mu}}
$$

from (37) for consideration of (28). And we can rewrite (38) by using (21) for

$$
A_{, v}^{\mu}=\frac{\partial A^{\mu}}{\partial x^{v}} .
$$

Because the second term of the right side of (38) does not exist here, we may adopt (38) and (39) description form of which. Well, I get conclution from (39), Definition 12 that if all coordinate systems $x^{\mu}, x^{v}, x^{\sigma}, x^{\lambda}, \cdots$ satisfied (5), Convariant differentiation for Contravariant Bector $A_{, v}^{\mu}$ behave like a Convariant differentiation for Scalar $S_{; v}$.

-End Proof 


\section{Discussion}

About Definition 2:

I named (5) "Binary Law" by Proposition 3.

About Proposition 6:

Convariant and contravariant tensor of the first rank don't change the formula whether it's satisfied (5) or not.

About Proposition 8:

In (32), we can think that $\frac{\partial A_{\mu}}{\partial x_{\mu}}$ expressed the distance of two points in $\frac{\partial g^{\nu \mu}}{\partial x^{\mu}}=0$ is establishment and this is constant. And, $A_{\mu}^{\mu}$; expresses the distance of two points in general and this is not constant.

About Proposition 9:

In (39), we can handle $\frac{\partial A^{\mu}}{\partial x^{v}}$ as tensor similarly $A_{, v}^{\mu}$.

\section{References}

[1] Einstein, A. (1916) Die Grundlage der allgemeinen Relativitätstheorie. Annalen der Physik, $49,769$.

[2] Dirac, P.A.M. (1975) General Theory of Relativity. John Wiley and Sons, Inc., Hoboken.

Submit or recommend next manuscript to SCIRP and we will provide best service for you:

Accepting pre-submission inquiries through Email, Facebook, LinkedIn, Twitter, etc.

A wide selection of journals (inclusive of 9 subjects, more than 200 journals)

Providing 24-hour high-quality service

User-friendly online submission system

Fair and swift peer-review system

Efficient typesetting and proofreading procedure

Display of the result of downloads and visits, as well as the number of cited articles

Maximum dissemination of your research work

Submit your manuscript at: http://papersubmission.scirp.org/

Or contact jmp@scirp.org 